\title{
Diagnóstico das práticas de alimentação complementar para o matriciamento das ações na Atenção Básica
}

\author{
Diagnosis of complementary feeding practices \\ for creating a matrix model for action in primary health care
}

Laís Amaral Mais ${ }^{1}$

Semíramis Martins Álvares Domene ${ }^{2}$

Marina Borelli Barbosa ${ }^{1}$

José Augusto de Aguiar Carrazedo Taddei ${ }^{1}$

'Departamento de

Pediatria, Universidade Federal de São Paulo. R.

Loefgreen 1647, Vila

Mariana. 04.040-032 São

Paulo SP Brasil.

lais.amaral88@yahoo.com.br

${ }^{2}$ Departamento de Políticas

Públicas e Saúde Coletiva,

Instituto Saúde e Sociedade,

Universidade Federal de São

Paulo.
Abstract Timely and appropriate complementary feeding is essential for the healthy growth and development of children, and Primary Health Care, especially the Family Health Support Nuclei, are the ideal location for developing relevant actions during this period. A cross-sectional study that applied a questionnaire to mothers and anthropometric evaluation for 324 children sought to develop an index of complementary feeding inadequacies and to study its association with social, economic, clinical, epidemiological and nutritional variables. For quantification of feeding inadequacies, an index using the Delphi method was created. High frequencies were observed for all inadequacies, especially for late introduction of solids (80.2\%), early introduction of sugar/thickeners $(78.1 \%)$ and liquids (73.5\%). The most significant results of these associations were early weaning of exclusive $(p=0.000)$ and total $(p=$ $0.005)$ breastfeeding, absence of partner $(p=0.001)$ and the mother supporting the family financially $(p=0.025)$. The use of this index identifies higher-risk situations for developing a nutritional assistance action plan, especially when it comes to promoting matrix model work.

Key words Complementary feeding, Infant nutrition, Nutritionist; Primary Health Care
Resumo A alimentação complementar adequada e oportuna é essencial para o crescimento e o desenvolvimento saudáveis da criança, sendo a Atenção Primária à Saúde, em especial os Núcleos de Apoio à Saúde da Família, o lócus ideal para o desenvolvimento de ações pertinentes a esta prática. Estudo transversal com aplicação de questionário para mães e avaliação antropométrica de 324 crianças visou desenvolver um escore de inadequações na alimentação complementar e estudar suas relações com variáveis socioeconômicas, clínico-epidemiológicas e nutricionais. Para quantificação das inadequações alimentares foi criado um escore por meio do Método Delphi. Foram observadas altas frequências para todas as inadequações, especialmente na introdução tardia de sólidos $(80,2 \%)$, precoce de açúcares/engrossantes $(78,1 \%)$ e precoce de líquidos (73,5\%). Entre as variáveis mais significantemente associadas com o escore estão desmame precoce do aleitamento materno exclusivo $(p=0,000)$ e total $(p=0,005)$, ausência de companheiro $(p=0,001)$ e a mãe ser chefe da família $(p=0,025)$. A utilização do escore identifica situações de maior risco para subsidiar as ações prioritárias da assistência nutricional, especialmente para promover o trabalho matricial. Palavras-chave Alimentação complementar, Nutrição do lactente, Nutricionista, Atenção Primária à Saúde 


\section{Introdução}

A partir do sexto mês de vida da criança, a quantidade e a composição do leite materno não são suficientes para atender as necessidades nutricionais da criança, e tem início o período da alimentação complementar (AC). Nesta fase, os novos alimentos devem ser introduzidos de forma lenta e gradual, com consistência espessa e proveniente de todos os grupos alimentares, evitando-se os produtos industrializados ${ }^{1-3}$.

Nos últimos anos, as ações de aleitamento materno (AM) sofreram grandes avanços, porém o mesmo não ocorreu em relação à $\mathrm{AC}$, habitualmente iniciada precocemente e de forma inadequada, com predominância de alimentos lácteos, preparados à base de leite de vaca integral, acrescidos de farináceos e açúcar ${ }^{1,4-7}$.

Um dos motivos para este cenário é a dificuldade encontrada pelos profissionais de saúde frente à escassez de consensos e o contingenciamento de recursos destinados à formação continuada dos profissionais da rede e estruturação da Atenção Básica $(A B)^{8,9}$. Nesta direção, o governo federal publicou diversos documentos com estratégias voltadas à AC como a "ENPACS - Estratégia Nacional para Alimentação Complementar" (2010) e o programa "Amamenta e Alimenta Brasil", que reforça e incentiva a promoção do AM e da AC saudável no âmbito do Sistema Único de Saúde (SUS) 2,3,10,11.

No início dos anos 90 é lançada a Estratégia Saúde da Família (ESF), que tem como base a atenção em seu território, a partir de ações de promoção, proteção e recuperação da saúde desenvolvidas por uma equipe multidisciplinar, com ênfase na autonomia dos sujeitos, no planejamento local participativo e na parceria com os demais setores ${ }^{12,13}$. Como suporte técnico-pedagógico e retaguarda assistencial, é proposto o apoio matricial às Equipes de Saúde da Família (SF) a fim de ampliar seu campo de atuação e qualificar as ações em saúde, integrando distintas especialidades ${ }^{14-18}$.

Para estruturar o apoio matricial, em 2008 foram criados os Núcleos de Apoio à Saúde da Família (NASF), "com o objetivo de ampliar a abrangência e a resolutividade das ações da Atenção Primária (AP), apoiando a inserção da ESF na rede de serviços e o processo de territorialização e regionalização a partir da AP". O nutricionista é um dos profissionais que podem compor esta equipe ${ }^{19,20}$.

Entre as ações de alimentação e nutrição a serem desenvolvidas nos NASF pelas Equipes de
SF incluem-se a promoção de práticas alimentares saudáveis; a construção de estratégias voltadas aos distúrbios alimentares, o desenvolvimento de projetos terapêuticos, especialmente para doenças e agravos não transmissíveis; a realização do diagnóstico alimentar e nutricional da população, com o reconhecimento do território e a identificação de grupos de risco, e demais ações para a promoção da Segurança Alimentar e Nutricional (SAN) e do Direito Humano à Alimentação Adequada (DHAA) ${ }^{20}$.

A compreensão da realidade por meio do reconhecimento do território e dos indicadores epidemiológicos e nutricionais permite conhecer o contexto social e promover a corresponsabilização e o protagonismo dos sujeitos. A abordagem multiprofissional favorece o estabelecimento de associações entre as características culturais e sociodemográficas da família, seu padrão de vida e práticas alimentares inadequadas, como a introdução em tempo inoportuno da $\mathrm{AC}^{8,21}$.

A utilização de um escore construído a partir de variáveis socioeconômicas, clínico-epidemiológicas e nutricionais possibilita resumir em uma escala componentes complexos de um processo de determinação e tem como vantagens incorporar o conhecimento acumulado na literatura na exploração do processo em questão ao atribuir valor específico para cada indivíduo da população estudada, gerando informação de fácil compreensão e utilização $22-24$.

O objetivo deste trabalho foi desenvolver um escore de inadequações na AC (EIAC) e estudar sua associação com variáveis socioeconômicas, clínico-epidemiológicas e nutricionais. Esta análise visa propor ações e estratégias para a abordagem nutricional adequada da criança durante o primeiro ano de vida no âmbito da AP.

\section{Métodos}

Trata-se de um estudo transversal de abordagem quali-quantitativa que se deu a partir do contato com a equipe da Unidade de Saúde da Família Perseu Leite de Barros (USF-PLB), localizada no distrito Noroeste do município de Campinas, SP, e da formalização da parceria com a Secretaria Municipal de Saúde. As informações provenientes de um questionário realizado com famílias com crianças menores de 6 anos foram o objeto de análise.

Foi realizado o reconhecimento da área de abrangência da USF, nos anos de 2009 e 2010. Os dados do Censo de 2008 (IBGE) foram empre- 
gados para a realização da amostragem, que ocorreu de acordo com a distribuição da população nos domicílios da área de cobertura da USF referentes a 19 setores censitários. Foi sorteado em cada setor o número de residências proporcional à sua representatividade na área de estudo, seguida da seleção dos domicílios por critério de conveniência.

Os critérios de inclusão das unidades amostrais para o estudo foram famílias com crianças de zero a seis anos, não portadoras de doenças crônicas e residentes na área de cobertura da USF.

Para a realização da coleta de dados, três nutricionistas foram treinadas quanto à abordagem para aplicação do questionário junto às mães e às técnicas antropométricas para avaliação da condição nutricional das crianças. Utilizou-se estadiômetro horizontal para crianças menores de 2 anos, estadiômetro vertical para aquelas entre 2 a 6 anos e para ambas a balança digital, procedimentos realizados de acordo com as orientações da $\mathrm{OMS}^{25}$. As mensurações antropométricas foram classificadas segundo as curvas de crescimento da OMS de 2005, utilizando-se os programas WHO Anthro ${ }^{26}$ e WHO AnthroPlus ${ }^{27}$. As entrevistas com os responsáveis das crianças residentes nos domicílios selecionados e obedecendo a proporcionalidade amostral foram realizadas em três situações: na própria USF, enquanto os pacientes aguardavam na sala de espera pelas consultas; na creche local ou durante as visitas domiciliares, com o acompanhamento dos Agentes Comunitários de Saúde (ACS).

O questionário estruturado e pré-codificado utilizado continha questões sobre condições sociodemográficas (condição empregatícia e escolaridade materna, presença de companheiro, chefe da família, faixa de renda, tipo de moradia, apoio de programa do governo), de gestação ( $n^{\circ}$ de consultas pré-natal, tabagismo, tipo de parto, intervalo interpartal), de nascimento da criança (prematuridade, peso ao nascer, $n^{\circ}$ de irmãos), sobre AM (tempo de aleitamento materno total (AMT) e exclusivo (AME), idade de introdução da chupeta), introdução de alimentos (local e quem orientou a introdução da $\mathrm{AC}$, idades de introdução dos alimentos) e avaliação antropométrica [estatura/idade (E/I) e índice de massa corporal/idade (IMC/I)].

Foram entrevistadas 339 famílias, com 15 perdas por: dados incompletos do questionário; criança portadora de deficiência; criança adotada; famílias não residentes da área de cobertura da USF. Assim, a população de estudo foi composta por 324 crianças entre zero e seis anos com perda amostral de 4,4\%. Quando existia mais que uma criança menor de seis anos no domicílio que compunha a amostra, a análise foi restrita à última criança nascida de cada família para diminuir o viés recordatório e evitar a dupla representação da unidade amostral.

Foi criado um banco de dados no pacote estatístico SPSS $18.0^{28}$, com dupla digitação e validação dos dados e, para a quantificação das inadequações alimentares de cada criança foi criado o Escore de Inadequações na Alimentação Complementar (EIAC) com pontuação de zero a dez a partir de sete componentes de análise, conforme demonstrado na Tabela 1.

O EIAC foi desenvolvido a partir dos resultados de painéis de especialistas estruturados em consonância com o Método Delphi, que se fundamenta em pesquisas estruturais e utiliza de forma quantitativa as melhores estimativas ponderais dos participantes, os quais devem ser especialistas no tema em questão ${ }^{29}$. Para o desenvolvimento do EIAC se considerou a importância de cada componente de inadequação na alimentação da criança; assim quanto mais graves as consequências para a saúde da criança, maior a pontuação do componente. No caso da ocorrência de desvio de um ou mais itens de uma mesma inadequação, a pontuação não muda; foram consultados 10 especialistas, doutores na área e com experiência na $\mathrm{AB}$.

Para o estudo da associação entre o EIAC e as variáveis de interesse foi utilizado o modelo bivariado, definido a partir dos valores de $\mathrm{p}<0,1$ nos testes do qui-quadrado, justificados pela natureza do estudo qualiquantitativo. O ponto de corte utilizado para o EIAC foi $<$ P25 para menos inadequações e $\geq$ P25 para mais inadequações.

O estudo integrou o projeto 'Desenvolvimento de modelo matricial para a inserção de ações de nutrição e dietética para o combate aos principais distúrbios nutricionais no Programa de Saúde da Família - PSF-Nutri', financiado pela Fundação de Amparo à Pesquisa do Estado de São Paulo - FAPESP.

\section{Resultados}

A Tabela 2 contém os dados do estudo da associação entre as variáveis socioeconômicas, clínicoepidemiológicas e nutricionais e o EIAC. Segundo a análise descritiva, grande parte das mães apresentou baixa escolaridade $(38,3 \%)$ e, a maioria delas, baixa renda familiar $(71,1 \%)$, sendo que $76,5 \%$ estão empregadas. Apenas $22,5 \%$ das mães 
Tabela 1. Distribuição da ocorrência das inadequações alimentares que compõem o Escore de Inadequações na Alimentação Complementar (EIAC). Centro de Saúde Perseu Leite de Barros (CS-PLB), Campinas, SP, 2009-2010.

\begin{tabular}{|c|c|c|c|c|}
\hline $\begin{array}{l}\text { Inadequações } \\
\text { alimentares }\end{array}$ & $\begin{array}{c}\text { \% de ocorrência da } \\
\text { inadequação na amostra } \\
\qquad(\mathrm{n}=324)\end{array}$ & $\begin{array}{l}\text { Ponte de } \\
\text { corte }\end{array}$ & Composição & $\begin{array}{c}\text { Valor para } \\
\text { ponderação } \\
\text { do EIAC }\end{array}$ \\
\hline $\begin{array}{l}\text { Introdução precoce } \\
\text { de líquidos }\end{array}$ & 73,5 & $<6$ meses & $\begin{array}{l}\text { Água } \\
\text { Chá } \\
\text { Suco de frutas }\end{array}$ & 1,25 \\
\hline $\begin{array}{l}\text { Introdução precoce } \\
\text { de sólidos }\end{array}$ & 48,1 & $<8$ meses & $\begin{array}{l}\text { Papa de frutas } \\
\text { Papa salgada } \\
\text { Carne vermelha } \\
\text { Frango } \\
\text { Fígado } \\
\text { Peixe } \\
\text { Cereal } \\
\text { Leguminosa } \\
\text { Alimentação da família }\end{array}$ & 2 \\
\hline $\begin{array}{l}\text { Introdução precoce } \\
\text { de leite de vaca }\end{array}$ & 39,8 & $<12$ meses & & 2 \\
\hline $\begin{array}{l}\text { Introdução precoce } \\
\text { de açúcar e/ou } \\
\text { engrossante }\end{array}$ & 78,1 & $<12$ meses & & 1,5 \\
\hline $\begin{array}{l}\text { Introdução tardia de } \\
\text { líquidos }\end{array}$ & 19,8 & $>7$ meses & $\begin{array}{l}\text { Água } \\
\text { Suco de frutas }\end{array}$ & 0,5 \\
\hline $\begin{array}{l}\text { Introdução tardia de } \\
\text { sólidos }\end{array}$ & 80,2 & $>12$ meses & $\begin{array}{l}\text { Papa de frutas } \\
\text { Papa salgada } \\
\text { Carne vermelha } \\
\text { Frango } \\
\text { Fígado } \\
\text { Peixe } \\
\text { Cereal } \\
\text { Leguminosa } \\
\text { Alimentação da família }\end{array}$ & 1,5 \\
\hline $\begin{array}{l}\text { Inadequação no } \\
\text { preparo de papa }\end{array}$ & 57,1 & & $\begin{array}{l}\text { Utilização de outras } \\
\text { gorduras (exceto óleo) } \\
\text { Utilização de condimentos } \\
\text { industrializados } \\
\text { Total }\end{array}$ & 1,25 \\
\hline
\end{tabular}

vivem sem companheiro, mas 19,5\% do total são as principais provedoras da casa, caracterizandose como chefes da família. A minoria da população não possui casa própria $(36,7 \%)$, bem como recebe apoio de programa do governo $(28,1 \%)$.

Em relação às características de gestação e nascimento da criança, 69,9\% das mães realizaram 8 ou mais consultas pré-natal, porém mais da metade dos partos foram cesáreas $(54,6 \%)$, provavelmente o que justifica os índices elevados de prematuridade e de baixo peso ao nascer, $10,5 \%$ e 10,2\%, respectivamente. Quanto à antropometria, desvios tanto de E/I quanto de IMC/I foram observados em $7,1 \%$ e $20,7 \%$ das crianças, respectivamente.

O local onde a maioria das crianças iniciou a AC foi na própria casa $(95,6 \%)$ e normalmente com orientação do pediatra $(57,9 \%)$. Alta frequência foi observada na introdução precoce da chupeta $(69,8 \%)$. 
Tabela 2. Distribuição das variáveis socioeconômicas, clínico-epidemiológicas e nutricionais segundo categorias do Escore de Inadequações Alimentares (EIAC). Centro de Saúde Perseu Leite de Barros (CS-PLB), Campinas, SP, 2009-2010.

\begin{tabular}{|c|c|c|c|c|c|}
\hline Variáveis & $\begin{array}{c}\mathbf{n} \\
\text { (\% categoria } \\
\text { de risco) }\end{array}$ & $\begin{array}{c}\text { Mais } \\
\text { inadequações } \\
(\%)\end{array}$ & $\begin{array}{c}\text { Menos } \\
\text { inadequações } \\
(\%)\end{array}$ & $\begin{array}{l}\text { Odds Ratios } \\
\text { (IC 90\%) }\end{array}$ & $\mathbf{p}$ \\
\hline Escolaridade materna & $324(38,3)$ & & & $0,93(0,53-1,63)$ & 0,797 \\
\hline$\leq 8$ anos & & $79,8(99 / 124)$ & $20,2(25 / 124)$ & & \\
\hline$>8$ anos & & $81,0(162 / 200)$ & $19,0(38 / 200)$ & & \\
\hline Desemprego da mãe & $217(23,5)$ & & & $0,77(0,36-1,68)$ & 0,509 \\
\hline Desempregada & & $78,4(40 / 51)$ & $21,6(11 / 51)$ & & \\
\hline Empregada & & $82,5(137 / 166)$ & $17,5(29 / 166)$ & & \\
\hline Presença de companheiro & $324(22,5)$ & & & $5,30(1,85-15,14)$ & 0,001 \\
\hline Não & & $94,5(69 / 73)$ & $5,5(4 / 73)$ & & \\
\hline Sim & & $76,5(192 / 251)$ & $23,5(59 / 251)$ & & \\
\hline Chefe da família & $308(19,5)$ & & & $2,89(1,10-7,60)$ & 0,025 \\
\hline Mãe & & $91,7(55 / 60)$ & $8,3(5 / 60)$ & & \\
\hline Outro & & $79,2(194 / 245)$ & $20,8(51 / 245)$ & & \\
\hline Faixa de renda & $322(71,1)$ & & & $0,70(0,37-1,36)$ & 0,291 \\
\hline Abaixo da linha de pobreza & & $79,0(181 / 229)$ & $21,0(48 / 229)$ & & \\
\hline Acima da linha de pobreza & & $84,3(75 / 89)$ & $15,7(14 / 89)$ & & \\
\hline Tipo de moradia & $324(63,3)$ & & & $1,61(0,93-2,83)$ & 0,088 \\
\hline Própria & & $83,4(171 / 205)$ & $16,6(34 / 205)$ & & \\
\hline Não própria & & $75,6(90 / 119)$ & $24,4(29 / 119)$ & & \\
\hline Apoio de programa de governo & $324(28,1)$ & & & $1,47(0,76-2,81)$ & 0,249 \\
\hline Não & & $84,6(77 / 91)$ & $15,4(14 / 91)$ & & \\
\hline Sim & & $79,0(184 / 233)$ & $21,0(49 / 233)$ & & \\
\hline No de consultas pré-natal & $288(30,9)$ & & & $1,95(0,95-3,99)$ & 0,066 \\
\hline$\leq 8$ consultas & & $87,6(78 / 89)$ & $12,4(11 / 89)$ & & \\
\hline$>8$ consultas & & $78,5(153 / 195)$ & $21,5(42 / 195)$ & & \\
\hline Tabagismo na gestação & $324(13,3)$ & & & $1,07(0,47-2,42)$ & 0,881 \\
\hline Sim & & $81,4(35 / 43)$ & $18,6(8 / 43)$ & & \\
\hline Não & & $80,4(226 / 281)$ & $19,6(55 / 281)$ & & \\
\hline Tipo de parto & $324(54,6)$ & & & $1,03(0,60-1,80)$ & 0,906 \\
\hline Cesárea & & $80,8(143 / 177)$ & $19,2(34 / 177)$ & & \\
\hline Normal & & $80,3(118 / 147)$ & $19,7(29 / 147)$ & & \\
\hline Intervalo interpartal & $147(9,5)$ & & & $0,79(0,20-3.05)$ & 0,730 \\
\hline$<2$ anos & & $78,6(11 / 14)$ & $21,4(3 / 14)$ & & \\
\hline$\geq 2$ anos & & $82,3(107 / 130)$ & $17,7(23 / 130)$ & & \\
\hline Prematuridade & $320(10,5)$ & & & $1,37(0,51-3,71)$ & 0,530 \\
\hline$<37$ semanas & & $85,3(29 / 34)$ & $14,7(5 / 34)$ & & \\
\hline$\geq 37$ semanas & & $80,9(228 / 282)$ & $19,1(54 / 282)$ & & \\
\hline Peso de nascimento & $324(10,2)$ & & & $1,41(0,52-3,80)$ & 0,500 \\
\hline$<2500 \mathrm{~g}$ & & $84,8(28 / 33)$ & $15,2(5 / 33)$ & & \\
\hline$\geq 2500 \mathrm{~g}$ & & $79,9(231 / 289)$ & $20,1(58 / 289)$ & & \\
\hline No de irmãos da criança & $324(57,7)$ & & & $1,21(0,70-2,10)$ & 0,502 \\
\hline 1 ou mais irmãos & & $81,8(153 / 187)$ & $18,2(34 / 187)$ & & \\
\hline Sem irmãos & & $78,8(108 / 137)$ & $21,2(29 / 137)$ & & \\
\hline
\end{tabular}

Observou-se que as inadequações em relação à introdução precoce de alimentos ocorrem na maioria dos casos. A maioria das crianças $(36,8 \%)$ recebeu a $\mathrm{AC}$ por crenças da mãe e de parentes, por entenderem que o tempo era opor- tuno ou que somente o leite materno não era suficiente; $32,4 \%$ dos pais introduziram os novos alimentos por orientação do pediatra. Já a volta ao trabalho da mãe e/ou a entrada da criança na creche foram responsáveis pela introdução da 
Tabela 2. continuação

\begin{tabular}{|c|c|c|c|c|c|}
\hline Variáveis & $\begin{array}{c}\mathbf{n} \\
(\% \text { categoria } \\
\text { de risco })\end{array}$ & $\begin{array}{c}\text { Mais } \\
\text { inadequações } \\
(\%)\end{array}$ & $\begin{array}{c}\text { Menos } \\
\text { inadequações } \\
(\%)\end{array}$ & $\begin{array}{l}\text { Odds Ratios } \\
\text { (IC 90\%) }\end{array}$ & $\mathbf{p}$ \\
\hline Altura/idade da criança & $324(7,1)$ & & & $1,16(0,38-3,53)$ & 0,796 \\
\hline Baixa estatura $(<-2 \mathrm{DP})$ & & $82,6(19 / 23)$ & $17,4(4 / 23)$ & & \\
\hline Estatura adequada $(\geq-2 \mathrm{DP})$ & & $80,4(242 / 301)$ & $19,6(59 / 301)$ & & \\
\hline IMC/idade da criança & $324(20,7)$ & & & $1,14(0,57-2,28)$ & 0,722 \\
\hline Desvio nutricional & & $82,1(55 / 67)$ & $17,9(12 / 67)$ & & \\
\hline Eutrofia & & $80,2(206 / 257)$ & $19,8(51 / 257)$ & & \\
\hline Tempo de aleitamento materno total & $230(48,7)$ & & & $3,08(1,37-6,93)$ & 0,005 \\
\hline$<8$ meses & & $92,0(103 / 112)$ & $8,0(9 / 112)$ & & \\
\hline$\geq 8$ meses & & $78,8(93 / 118)$ & $21,2(25 / 118)$ & & \\
\hline Tempo de aleitamento materno exclusivo & $309(78,3)$ & & & $4,51(2,45-8,28)$ & 0,000 \\
\hline$<6$ meses & & $86,0(208 / 242)$ & $14,0(34 / 242)$ & & \\
\hline$\geq 6$ meses & & $57,6(38 / 66)$ & $42,4(28 / 66)$ & & \\
\hline Local de início da introdução de alimentos & $322(95,6)$ & & & $0,32(0,04-2,48)$ & 0,249 \\
\hline Casa & & $80,5(248 / 308)$ & $19,5(60 / 308)$ & & \\
\hline Creche e outros & & $92,9(13 / 14)$ & $7,1(1 / 14)$ & & \\
\hline Quem orientou a introdução dos alimentos & $321(57,9)$ & & & $0,73(0,41-1,31)$ & 0,292 \\
\hline Pediatra & & $79,0(147 / 186)$ & $21,0(39 / 186)$ & & \\
\hline Outros & & $83,7(113 / 135)$ & $16,3(22 / 135)$ & & \\
\hline Idade de introdução da chupeta & $139(69,8)$ & & & $0,85(0,25-2,83)$ & 0,785 \\
\hline$<1$ mês & & $88,7(86 / 97)$ & $11,3(11 / 97)$ & & \\
\hline$\geq 1$ mês & & $90,2(37 / 41)$ & $9,8(4 / 41)$ & & \\
\hline
\end{tabular}

* Teste do qui-quadrado $(\mathrm{p}<0,1)$.

AC em 12,5\% dos casos, percentual similar às questões relacionadas à criança e suas necessidades, como a própria vontade do bebê, cólica, sede e como forma de acalmar a criança $(10,1 \%)$. Outros motivos levaram a $8,1 \%$ da introdução de alimentos.

O estudo analítico para verificação da associação entre as variáveis indica que a ausência de companheiro ( $\mathrm{OR}=5,30$ e $\mathrm{IC}=1,85-15,14)$, a mãe ser chefe da família $(\mathrm{OR}=2,89$ e IC $=1,10$ $7,60)$ e possuir moradia própria $(\mathrm{p}=0,088, \mathrm{OR}$ $=1,61$ e IC $=0,93-2,83$ ) estão relacionados a maiores pontuações no EIAC. Ter realizado 8 consultas pré-natal ou menos também se revelou uma variável de risco quando associada ao EIAC $(\mathrm{p}=0,066, \mathrm{OR}=1,95$ e IC $=0,95-3,99)$.

Também houve associação entre o EIAC e o menor tempo AMT $(\mathrm{OR}=3,08$ e $\mathrm{IC}=1,37-6,93)$ e $\mathrm{AME}(\mathrm{OR}=4,51$ e $\mathrm{IC}=2,45-8,28)$, por se tratar de variáveis espelhares da introdução da AC.

\section{Discussão}

O presente estudo é de caráter operacional e descreve um método para a sistematização de dados relevantes para o planejamento de ações que visam o matriciamento em nutrição infantil no âmbito da ESF, principal orientação metodológica adotada na política nacional de saúde voltada à $\mathrm{AB}$ e ao fortalecimento da rede de assistência; adota o processo de avaliação de situações de determinação complexa a partir da adoção de escore produzido por meio de painéis de especialistas como estratégia inovadora para instrumentalizar o trabalho matricial dos 1038 nutricionistas presentes nos 1388 NASF implantados até julho de $2011^{30}$.

Os resultados apresentados neste estudo podem ser adotados em unidades e agrupamentos populacionais similares, sobretudo para ações de monitoramento. Não se constituem, no entanto, em informações referentes a processos biológicos e fisiopatológicos, mas ligados à organização social e política das comunidades, podendo, portanto, variar em diferentes cenários. Isso justifica a proposta de replicação do método para orientação das ações de nutrição infantil visando à geração de informações que poderão contemplar a ampla gama de situações encontradas na ESF na forma de novos componentes de análise.

No modelo de política de saúde adotado no Brasil, a Atenção Primária à Saúde (APS) tem os 
NASF como apoio da ESF, organizados a partir de ferramentas conceituais como o apoio matricial que orienta os processos de trabalho ${ }^{21}$. A proposta de ações deriva do reconhecimento do território, que levou ao diagnóstico das práticas de AC na população estudada. Para isso, foram utilizadas as frequências dos desvios alimentares, os motivos que levaram as mães a introduzir precocemente os alimentos e o EIAC associado a variáveis socioeconômicas, clínico-epidemiológicas e nutricionais das mães e de seus filhos.

Os resultados disponíveis a partir do reconhecimento do território vão ao encontro dos dados apresentados na literatura para subsidiar as propostas de orientação e operacionalização na alimentação infantil no âmbito do NASF. Um exemplo são as frequências de inadequações alimentares apresentadas na Tabela 1. Destaca-se que $73,5 \%$ das crianças receberam precocemente algum tipo de líquido (água, chá, suco de frutas), $39,8 \%$ receberam leite de vaca e $78,1 \%$ receberam açúcar e/ou engrossante antes dos 12 meses. Tais resultados são concordantes com aqueles encontrados em estudos transversais com aplicação de questionário realizados em cidades de grandes centros urbanos brasileiros ${ }^{31-33}$.

Acredita-se que o motivo para a introdução precoce de água e suco de frutas se dá pela crença das mães de que a criança sente sede e pode desidratar-se, principalmente em períodos de calor; quanto ao chá, atribui-se a este alimento propriedades calmantes e de melhora de episódios de cólica, o que não encontra apoio nos estudos a este respeito ${ }^{34-36}$.

Já a introdução precoce de leite de vaca integral ocorre habitualmente por ser uma característica cultural, já que é visto como um dos alimentos mais importantes para a saúde da criança $^{37}$. Sua introdução antes dos 12 meses é contraindicada por ser responsável por $20 \%$ das alergias alimentares, podendo desencadear diabetes melito tipo 1, doenças atópicas como a asma, micro-hemorragias intestinais, e sobrecarga do sistema imune e renal, bem como o desmame precoce $^{6,38}$.

No Brasil esse desvio alimentar ocorre com a introdução combinada de açúcares e engrossantes, alimentos que além de serem isentos de micronutrientes, aumentam a densidade energética e tem em sua composição a sacarose, considerada o dissacarídeo mais cariogênico. Tais alimentos são precocemente introduzidos na alimentação infantil pela tendência materna de oferecer alimentos doces, o que "satisfará o paladar da criança" e "a manterá bem alimentada"1,39,40.
Em relação ao oferecimento precoce de sólidos em $48,1 \%$ dos casos, houve concordância com os achados de estudos nacionais e internacionais $^{31,35,41}$. Dentre as desvantagens da introdução precoce de sólidos estão a diminuição da duração do AM e a interferência na absorção de nutrientes importantes existentes no leite humano, como ferro e zinco ${ }^{42}$. Segundo estudo qualitativo realizado em cinco macrorregiões brasileiras, a percepção da fome é a lógica que leva as mães a precipitarem a $\mathrm{AC}^{43}$.

A introdução tardia da AC também se constitui em importante erro alimentar pelo possível comprometimento de crescimento e desenvolvimento da criança, o que pode levar ao aumento do risco de desnutrição e deficiência de micronutrientes ${ }^{10,44}$. No presente estudo, $19,8 \%$ e $80,2 \%$ das crianças tiveram o primeiro contato com líquidos e sólidos tardiamente, de forma semelhante ao constatado por estudos realizados em São Paulo, SP e Florianópolis, SC $7,33,35$.

Outro aspecto observado na composição do EIAC foi a inadequação no preparo de papas (uso de condimentos industrializados e qualquer outra fonte de gordura que não seja o óleo vegetal), relatada por $57,1 \%$ dos responsáveis. Estudo argentino registrou que $76,09 \%$ dos responsáveis entrevistados adicionaram óleo vegetal à primeira papa salgada da criança, enquanto que $25,42 \%$ adicionaram manteiga ${ }^{45}$. De acordo com orientações do Ministério da Saúde (MS) ${ }^{46}$, é contraindicada a adição de condimentos prontos no preparo da papa salgada para evitar os riscos de excesso de sódio. Além disso, a fonte de gordura indicada é o óleo vegetal para fornecer ácidos graxos essenciais, além de promover melhor consistência e sabor aos alimentos ${ }^{2,47}$.

Os resultados apresentados na Tabela 2 mostram que as mães que vivem sem companheiro apresentam alto risco para cometer inadequações na AC, o que já foi observado na Austrália, onde $53,8 \%$ das mães sem companheiros introduziram a AC precocemente (antes das 17 semanas). Em Campinas, SP, encontrou-se risco seis vezes maior de mães sem companheiro amamentarem exclusivamente por menos tempo seus filhos. No mesmo município, a mediana de AME foi de 90 dias para as mães com companheiro, enquanto para as demais foi de apenas 60 dias, o que é espelhar para a introdução precoce de alimentos complementares, mais frequente entre mães que não contam com o suporte do parceiro ${ }^{5,32,48}$.

Em relação à associação encontrada entre o EIAC e a mãe ser chefe da família, não se encontrou estudo que utilize tal variável para explicar o 
grau de inadequações na introdução da AC. A mãe ser chefe da família relaciona-se com a ausência de companheiro, o que leva a mulher a trabalhar fora de casa em busca de meios para sustentar sua família, sendo esta, muitas vezes, a única fonte de renda da casa ${ }^{49}$.

A industrialização e a inserção da mulher no mercado de trabalho levam a alterações nos hábitos alimentares familiares e impactam diretamente na alimentação infantil ${ }^{32,50}$. Este cenário é característico da família contemporânea ${ }^{33}$. A responsabilidade pela renda familiar também interfere no aleitamento materno, como apontado em coorte realizada na capital paulista: as mães que não trabalhavam fora de casa apresentaram uma introdução mais tardia de outros tipos de leite e, portanto, maior tempo de aleitamento materno ${ }^{35}$. Estudo com 516 mães da cidade de Florianópolis encontrou associação entre baixa escolaridade materna e atividade fora do lar com introdução precoce de alimentos ${ }^{33}$.

A associação entre as maiores pontuações do EIAC e a realização de menor número de consultas durante o pré-natal também foi encontrada em inquérito domiciliar de abrangência nacional realizado na Índia e no Nepal, especificamente para os riscos de não introdução da AC, de baixa diversidade da dieta, de baixa frequência das refeições e de baixa aceitabilidade da dieta por parte da criança ${ }^{51,52}$.

A questão do AME e AMT relacionados a maiores valores de EIAC pode ser explicado pelo fato de o $\mathrm{AM}$ e a AC serem variáveis espelhares. Uma vez que a mãe ofereceu qualquer alimento à criança, o AME chega ao fim, o que provavelmente levará a um tempo de AMT menor, já que a criança começou a se alimentar com outros produtos e, muitas vezes, com outros tipos de leite ${ }^{5}$.

O EIAC foi criado pela escassez de indicadores consistentes que descrevam, meçam e quantifiquem as complexas práticas relacionadas à $\mathrm{AC}$; traduzidas na forma de um valor para estimar a inadequação da alimentação infantil, que é multidimensional, podem constituir um indicador de risco nutricional em saúde. No presente estudo, o objetivo foi resumir no EIAC as idades de introdução dos alimentos e o modo de preparo da papa salgada, na forma de um único indicador para facilitar a análise das associações. É importante enfatizar a falta de recomendações internacionais claras sobre a AC, o que dificulta o desenvolvimento de indicadores universais e de pontos de corte específicos ${ }^{22-24}$.

O EIAC tem o potencial de facilitar o planejamento de programas e de servir como ferramen- ta de monitoramento por meio da identificação de práticas alimentares inadequadas e de grupos vulneráveis, a partir da criação de programas de educação nutricional mais efetivos e da verificação do impacto dos programas já existentes ${ }^{24}$. Nos NASF, o EIAC funcionaria como um indicador para identificar as situações de maior risco para uma AC inadequada. Tais informações poderiam ser coletadas por profissionais das Equipes de SF, como os ACS, que estão presentes na USF em tempo integral. Após a coleta, os dados podem ser adotados pelo nutricionista do NASF para a avaliação da situação de saúde e nutrição daquela população, bem como decisões acerca da gestão do serviço de saúde ${ }^{53}$.

Este não é o primeiro escore criado para traduzir as práticas alimentares infantis. Em 2006, na Índia, foi criado o Infant and Child Feeding Index (ICFI) e foi estudada sua associação com o crescimento de crianças entre 6 e 23 meses. Este escore conta com cinco variáveis, sendo uma relativa ao tempo de introdução da AC, assim como o do presente estudo, dada a importância que a introdução oportuna dos alimentos tem sobre o crescimento e desenvolvimento infantis; os demais componentes, contudo, tratam da diversidade da dieta, da duração do aleitamento, do apoio psicossocial durante a alimentação e de aspectos relativos à higiene ${ }^{22,24}$.

Grande parte dos motivos citados pelas mães para a introdução precoce da AC está relacionada às suas crenças $(36,8 \%)$ ou de parentes, e à criança e suas necessidades $(10,1 \%)$. Tal fato é concordante com o estudo realizado em $2009^{48}$, cujos achados mostram que o principal motivo apontado pelas mães para introduzir sólidos antes das 17 semanas foi a percepção de que seus filhos precisavam ou estavam prontos para receber este tipo de alimento. Em ambos os casos os indicadores para tais motivos são subjetivos. No relato de uma mãe participante do estudo de Salve e Silva ${ }^{54}$, percebe-se claramente a influência direta que as crenças e percepções maternas têm sobre a alimentação infantil: "Uso a minha experiência, porque já cuidei muito de criança. É natural da vida, está com fome você dá comida. Quando você está comendo perto dela parece que come com os olhinhos, abre a boca, fica com vontade...”.

Por outro lado, $32,4 \%$ das mães relataram ter tomado a decisão de introduzir a $\mathrm{AC}$ com base nas condutas do pediatra; importante registrar que estas mães praticaram o desmame precoce associado a níveis insatisfatórios de saúde dos lactentes ${ }^{10}$. A falta de preparo e capacitação dos profissionais de saúde em relação ao tema 
justifica a necessidade de treinamentos e educação continuada, especialmente nas USF, onde existe a possibilidade do trabalho em conjunto de pediatras e nutricionistas por meio dos NASF ${ }^{5}$.

A necessidade de a mãe voltar ao trabalho para ajudar no sustento da família e assim ter que colocar a criança na creche, foi o motivo apontado por $12,5 \%$ das mães, frequência menor do que os $28,6 \%$ encontrados em estudo realizado em São Paulo, $\mathrm{SP}^{5}$; esta condição gera a necessidade de articular as responsabilidades do trabalho com as familiares, e a mulher assume as responsabilidades de manutenção e sustento econômico, afetivo, de cuidado dos filhos e da casa ${ }^{55}$.

Os achados deste estudo referentes à AC podem ter aplicabilidade nos processos de trabalho das Equipes de SF e dos NASF na definição e reconhecimento do território de atuação, a partir da análise da situação de saúde da população, considerando os aspectos culturais, sociais, econômicos, demográficos e epidemiológicos ${ }^{53}$. A atuação do nutricionista no NASF está em construção e ganha estrutura a partir das experiências de desenvolvimento e amadurecimento organizacional da assistência em rede. Algumas ações testadas por ocasião deste trabalho de campo mostraram-se efetivas e bem aceitas, tanto pela equipe quanto pela população.

Foram realizados grupos de gestantes interdisciplinares com a participação de ACS, enfermeiras e médicos, abordando questões relacionadas à alimentação da gestante e AM. Os resultados do EIAC poderiam fomentar, nesses grupos, a discussão de temas relacionados à AC.

Com o mesmo foco, porém de forma individualizada, foram realizados atendimentos com duração média de 20 minutos durante o pré-natal, abordando os temas 'alimentação na gestação e preparo das mamas' e 'AM' em diferentes trimestres. Por ser uma atividade individualizada, é de interesse abordar aquelas mães identificadas como mais vulneráveis pela pontuação do EIAC (mães sem companheiro, chefes da família e com casa própria), para abordagem do tema 'AC'.

Visando complementar o grupo de gestantes e o atendimento nutricional no pré-natal, foram realizadas visitas domiciliares às puérperas e aos recém-nascidos a fim de acompanhar as práticas de AM e de introdução oportuna dos alimentos. Este momento também é individualizado, portanto, pode ser priorizado quando identificada a situação de risco nutricional, para abordagem em visita com o acompanhamento de um ACS, profissional responsável pelo vínculo com a população ${ }^{56}$.
Outra ação que se mostrou simples, porém facilitadora do trabalho do nutricionista foi a adição de questões referentes ao estado nutricional da criança e das práticas alimentares no questionário já utilizado pelos ACS para identificar os domicílios e as famílias residentes na área de cobertura da USF e Equipe de SF. Tais questões foram pensadas para serem objetivas, em número reduzido, mas que ofereçam ao nutricionista informações importantes para a identificação de situações de risco e aquelas já instaladas. À luz dos resultados das associações com o EIAC, é possível identificar mulheres grávidas mais propensas a cometerem inadequações na AC que, em uma percepção matricial na atenção, devem ser motivo de priorização das atividades coletivas comunitárias e da atuação da equipe.

A fim de se trabalhar com os profissionais da equipe, pode-se discutir o significado do EIAC e sua contribuição para o agendamento de visitas domiciliares e convite a participar de grupos. $\mathrm{O}$ escore é um instrumento pedagógico e facilitador da comunicação na equipe, a ser trabalhado por meio da educação nutricional a partir da problematização e contextualização dos casos vistos na comunidade.

As atividades educativas também foram realizadas na creche e na igreja locais. Naquele caso, os professores puderam aprender e tirar dúvidas sobre AM e AC, assuntos importantes visto que todas as crianças realizam pelo menos três refeições por dia na creche sob acompanhamento destes profissionais, potenciais estimuladores da alimentação saudável desde os primeiros anos de vida das crianças ${ }^{57}$. A aplicação do EIAC nesse contexto facilitaria a identificação de ações nas creches que inadvertidamente levam a inadequações na AC. Já na igreja local foram realizadas oficinas culinárias contando com a participação ativa das mães no preparo dos alimentos, o que foi estimulador especialmente para aquelas identificadas como vulneráveis pelo EIAC.

Ação promissora ainda não testada no âmbito do trabalho de campo é a realização de consultas compartilhadas. A criação de protocolos de atendimento com questões específicas sobre AC podem ser de grande valor ${ }^{53}$.

O presente estudo apresenta algumas limitações. O EIAC é um indicador para traçar o perfil da população do estudo, porém não considera o tempo de introdução de alimentos industrializados, consistência/textura da papa oferecida, utensílio utilizado no oferecimento da alimentação, horários e fracionamento das refeições, quantidade dos alimentos, bem como modo de preparo 
da papa. É importante ressaltar a ausência do tempo de introdução do ovo na AC, tendo em vista a recente mudança nas recomendações que anteciparam sua oferta na AC de 12 meses para a partir dos 6 meses. Tal mudança, ainda hoje, leva a condutas diversas por parte dos profissionais, o que poderia gerar um viés na composição do EIAC.

Além disso, os dados obtidos neste estudo são provenientes da área de cobertura de apenas uma USF, não sendo generalizáveis para todos os municípios brasileiros. Apesar destas limitações, a intenção da coleta de dados foi realizar o reconhecimento do território, com a identificação dos desvios mais frequentes na $\mathrm{AC}$ e de qual é o perfil mais associado a tais inadequações, dado que as características da área geográfica e dos usuários não são estáticas ${ }^{53}$.

\section{Conclusão}

Indicadores de práticas alimentares podem ser usados para compor um escore para acompanhamento da AC como parte das ações desenvolvidas pelo nutricionista na $\mathrm{AB}$. Na perspectiva do trabalho matricial, ferramentas de apoio à assistência que viabilizem o reconhecimento do território podem representar um recurso adicional para a adequada abordagem das situações de risco nutricional.

\section{Colaboradores}

LA Mais e JAAC Taddei participaram da concepção, delineamento, análise e interpretação dos dados, redação e revisão crítica do artigo, e aprovação da versão a ser publicada. SMA Domene participou da concepção, delineamento, redação e revisão crítica do artigo, e aprovação da versão a ser publicada. MB Barbosa participou da concepção, delineamento e revisão crítica do artigo. 


\section{Referências}

1. ESPGHAN Committee on Nutrition. Medical position paper - Complementary feeding: A commentary by the ESPGHAN Committee on Nutrition. $J$ Pediatr Gastroenterol Nutr 2008; 46(1):99-110.

2. Brasil. Ministério da Saúde (MS). Secretaria de Atenção à Saúde. Departamento de Atenção Básica. Saúde da Criança: Nutrição infantil:aleitamento materno e alimentação complementar. Brasília: MS; 2009. (Série A. Normas e Manuais Técnicos, Cadernos de Atenção Básica, No 23)

3. Brasil. Ministério da Saúde (MS). Dez passos para uma alimentação saudável: Guia alimentar para menores de dois anos - Um guia para o profissional da saúde na atenção básica. 2a Edição. Brasília: MS; 2010. (Série A. Normas e Manuais Técnicos)

4. Oliveira LPM, Assis AMO, Pinheiro SMC, Prado MS, Barreto ML. Alimentação complementar nos primeiros dois anos de vida. Rev Nutr 2005; 18(4): 459-469.

5. Barbosa MB, Palma D, Domene SMA, Taddei JAAC, Lopez FA. Fatores de risco associados ao desmame precoce e ao período de desmame em lactentes matriculados em creches. Rev Paul Pediatr 2009; 27(3):272-281.

6. Dias MCAP, Freire LMS, Franceschini SC. Recomendações para alimentação complementar de crianças menores de dois anos. Rev Nutr 2010; 23 (3):475-485.

7. Golin CK, Toloni MHA, Longo-Silva G, Taddei JAAC. Erros alimentares na dieta de crianças frequentadoras de berçários em creches públicas no município de São Paulo, Brasil. Rev Pauli Pediatr 2011; 29(1):35-40.

8. Fernandez PMF, Voci SM, Kamata LH, Najas MS, Souza ALM. Programa Saúde da Família e as ações em nutrição em um distrito de saúde do município de São Paulo. Cien Saude Colet 2005; 10(3):749-755.

9. Bassichetto KC, Réa MF. Infant and young child feeding counseling: An intervention study. J Pediatr 2008; 84(1):75-82.

10. Brasil. Ministério da Saúde (MS). Dez passos para uma alimentação saudável para crianças brasileiras menores de dois anos. Brasília: MS; 2010. (Série A, Normas e Manuais Técnicos)

11. Brasil. Ministério da Saúde (MS). ENPACS - Estratégia Nacional para Alimentação Complementar Caderno do Tutor. Série A. Normas e Manuais Técnicos. Brasília: MS; 2010.

12. Rosa WAG, Labate RC. Programa Saúde da Família: A construção de um novo modelo de assistência. Rev Lat Am Enfermagem 2005; 13(3):1027-1034.

13. Shimizu HE, Rosales C. As práticas desenvolvidas no Programa Saúde da Família contribuem para transformar o modelo de atenção à saúde? Rev Bras Enferm 2009; 62(3):424-429.

4. Figueiredo MD. Saúde mental na Atenção Básica: um estudo hermenêutico-narrativo sobre o apoio matricial na rede SUS-Campinas (SP) [tese]. Campinas: Faculdade de Ciências Médicas; 2006.

15. Nascimento CC. Apoio matricial em saúde mental: possibilidades e limites no contexto da Reforma Psiquiátrica [tese]. São Paulo: Escola de Enfermagem; 2007.
16. Arona EC. Implantação do matriciamento nos serviços de saúde de Capivari. Saude Soc 2009; 18(Supl. 1):26-36.

17. Dimenstein M, Severo AK, Brito M, Pimenta AL, Medeiros V, Bezerra E. O apoio matricial em Unidades de Saúde da Família: experimentando inovações em saúde mental. Saude Soc 2009; 18(1):63-74.

18. Figueiredo MD, Campos RO. Saúde Mental na atenção básica à saúde de Campinas, SP: uma rede ou um emaranhado? Cien Saude Colet 2009; 14(1):129138.

19. Brasil. Portaria $n^{\circ} 154$, de 24 de janeiro de 2008 . Cria os Núcleos de Apoio à Saúde da Família NASF. Diário Oficial da União 2008; 24 jan.

20. Brasil. Ministério da Saúde (MS). Secretaria de Atenção à Saúde. Departamento de Atenção Básica. Coordenação-Geral da Política de Alimentação e Nutrição. Manual de orientação das ações de alimentação e nutrição nos Núcleos de Apoio à Saúde da Família. Brasília: MS; 2010. (Série A. Normas e Manuais Técnicos)

21. Brasil. Ministério da Saúde (MS). Secretaria de Atenção à Saúde. Departamento de Atenção Básica. Diretrizes do NASF: Núcleo de Apoio à Saúde da Família. Brasília: MS; 2010. (Série A. Normas e Manuais Técnicos, Cadernos de Atenção Básica, No 27)

22. Srivastava N, Sandhu A. Infant and child feeding index. Indian J Pediatr 2006, 73(9):760-770.

23. Srivastava N, Sandhu A. Index for measuring child feeding practices. Indian J Pediatr 2007; 74(4):363368.

24. Garg A, Chadha R. Index for measuring the quality of complementary feeding practices in rural India. J Health Popul Nutr 2009; 27(6):763-771.

25. World Health Organization (WHO). Physical Status: The Use and Interpretation of Anthropometry. Geneva: WHO; 1995. (Technical Report Series, No 854)

26. World Health Organization (WHO). WHO Anthro for personal computers manual: Software for assessing growth and development of the world's children. Geneva: WHO; 2007.

27. World Health Organization (WHO). WHO AnthroPlus for personal computers manual: Software for assessing growth of the world's children and adolescents. Geneva: WHO; 2009.

28. Statistical Package for the Social Science for Windows (SPSS) versão 18.0. Chicago: SPSS; 2000.

29. Wounderberg F. An evaluation of Delphi. Technol Forecast Soc Change 1991; 40:131-150.

30. Brasil. Ministério da Saúde (MS). Departamento de Atenção Básica e Saúde da Família. Brasília: MS; 2011.

31. Modesto SP, Devincenzi UM, Sigulem DM. Práticas alimentares e estado nutricional de crianças no segundo semestre de vida atendidas na rede pública de saúde. Rev Nutr 2007; 20(4):405-415.

32. Bernardi JLD, Jordão RE, Barros Filho AA. Alimentação complementar de lactentes em uma cidade desenvolvida no contexto de um país em desenvolvimento. Rev Panam Salud Publica 2009; 26(5):405411. 
33. Corrêa EN, Corso ACT, Moreira EAM, Kazapi IAM. Alimentação complementar e características maternas de crianças menores de dois anos de idade em Florianópolis (SC). Rev Paul Pediatr 2009; 27(3):258-264.

34. Audi CAF, Corrêa AMF, Latorre MRDO. Alimentos complementares e fatores associados ao aleitamento materno e ao aleitamento materno exclusivo em lactentes até 12 meses de vida em Itapira, São Paulo, 1999. Rev Bras Saude Mater Infant 2003; 3(1):85-93.

35. Simon VGN, Souza JMP, Souza SB. Introdução de alimentos complementares e sua relação com variáveis demográficas e socioeconômicas, em crianças no primeiro ano de vida, nascidas em Hospital Universitário do município de São Paulo. Rev Bras Epidemiol 2003; 6(1):29-38.

36. Parada CMGL, Carvalhaes MABL, Jamas MT. Práticas de alimentação complementar em crianças no primeiro ano de vida. Rev Lat Am Enfermagem 2007; 15(2):282-289.

37. Farias Junior G, Osório MM. Padrão alimentar de crianças menores de cinco anos. Rev Nutr 2005; 18(6):793-802.

38. Brunken GS, Silva SM, França GVA, Escuder MM, Venâncio SI. Fatores associados à interrupção precoce do aleitamento materno exclusivo e à introdução tardia da alimentação complementar no centro-oeste brasileiro. J Pediatr 2006; 82(6):445-451.

39. Biral AM, Taddei JAAC, Passoni DF, Palma D. Índice de cárie e práticas alimentares entre crianças de creches do município de São Paulo. Rev Nutr 2013; 26(1):37-48.

40. Toloni MHT, Longo-Silva G, Goulart RMM, Taddei JAAC. Introdução de alimentos industrializados e de alimentos de uso tradicional na dieta de crianças de creches públicas no município de São Paulo. Rev Nutr 2011; 24(1):61-70.

41. Nwaru BI, Erkkola M, Ahonen S, Kaila M, Haapala A, Kronberg-Kippilä C, Salmelin R, Veijola R, Ilonen J, Simell O, Knip M, Virtanen SM. Age at the introduction of solid foods during the first year and allergic sensitization at the age 5 years. Pediatr 2010; 125(50):50-59.

42. Gomes PTT. Práticas alimentares de crianças menores de um ano que compareceram na Segunda Etapa da Campanha Nacional de Vacinação no Postos de Saúde fixos da cidade de Guarapuava-PR, 2004 [tese]. Ribeirão Preto: Universidade de São Paulo, Universidade Estadual do Centro-Oeste do Paraná; 2005.

43. Victora CG, Knauth DR. Hábitos alimentares de crianças menores de 2 anos: um estudo multicêntrico sobre práticas alimentares atuais no Brasil. In: Anais da Reunião da Associação Brasileira de Antropologia; 2000; Brasília.

44. Junqueira JM, Navarro AM, Cintra RMGC, Dias LCGD. Padrão alimentar de crianças brasileiras menores de 2 anos: uma visão crítica. Rev Simbiologias 2008; 1(1):184-199.

45. Gatica CI, Méndez de Feu MC. Prácticas de alimentación em ninõs menores de 2 años. Arch Argent Pediatr 2009; 107(6):496-503.
46. Brasil. Ministério da Saúde (MS), Organização Pan Americana da Saúde (Representação do Brasil). Guia alimentar para crianças menores de 2 anos.Brasília: MS; 2002. (Série A. Normas e Manuais Técnicos, No 107)

47. Lacerda EMA, Accioly E, Costa VM, Faria IG. Práticas de Nutrição pediátrica. São Paulo: Editora Atheneu; 2002.

48. Scott JA, Binns CW, Graham KI, Oddy WH. Predictors of the early introduction of solid foods in infants: results of a cohort study. BMC Pediatr 2009; 9(60):1-9.

49. Pinto RMF, Micheletti FABO, Bernardes LM, Fernandes JMPA, Monteiro GV, Silva MLN, Barreira TMHM, Makhoul AP, Cohn A. Condição feminina de mulheres chefes de família em situação de vulnerabilidade social. Serv Soc Soc 2011; (105):167-79.

50. Silva LMP, Venâncio SI, Marchioni DML. Práticas de alimentação complementar no primeiro ano de vida e fatores associados. Rev Nutr 2010; 23(6):983-992.

51. Joshi N, Agho KE, Dibley MJ, Senarath U, Tiwari K. Determinants of inappropriate complementary feeding practices in young children in Nepal: secondary data analysis of Demographic and Health Survey 2006. Matern Child Nutr 2012; 8(Supl. 1):45-59.

52. Patel A, Pusdekar Y, Bodhoniya N, Borkar J, Agho KE, Dibley MJ. Determinants of inappropriate complementary feeding practices in young children in India: secondary analysis of National Family Health Survey 2005-2006. Mater Child Nutr 2012; 8(Supl. $1): 28-44$.

53. Brasil. Ministério da Saúde (MS). Secretaria de Atenção à Saúde. Departamento de Atenção Básica. $\mathrm{Ca}$ dernos de atenção básica: Diretrizes do NASF - Núcleo de Apoio à Saúde da Família. Brasília: MS; 2009. (Série A - Normas e Manuais Técnicos, No 27)

54. Salve JM, Silva IA. Representações sociais de mães sobre a introdução de alimentos complementares para lactentes. Acta Paul Enferm 2009; 22(1):43-48.

55. Rotenberg S, Vargas S. Práticas alimentares e o cuidado da saúde: da alimentação da criança à alimentação da família. Rev Bras Saude Mater Infant 2004; 4(1):85-94.

56. Bachilli RG, Scavassa AJ, Spiri WC. A identidade do agente comunitário de saúde: uma abordagem fenomenológica. Cien Saude Colet 2008; 13(1):51-60.

57. Bernardon R, Silva JRM, Cardoso GT, Monteiro RA, Amorim NFA, Schmitz BAS, Rodrigues MLCF. Construção de metodologia de capacitação em alimentação e nutrição para educadores. Rev Nutr 2009; 22(3):389-398.

Artigo apresentado em 22/11/2012

Aprovado em 15/12/2012

Versão final apresentada em 26/12/2012 